\title{
MANGANESE NODULE DEEP SEA MINING
}

Thomas Bock, Günther Dörfler, Fritz Gehbauer, Werner Leyh Institut für Maschinenwesen im Baubetrieb

Universität Karlsruhe

Am Fasanengarten, 7500 Karlsruhe, Germany

\section{SUMMARY}

In cooperation with several French and German partners the Institut für Maschinenwesen im Baubetrieb is developing a manganese nodule mining system. The essential part of this mining system is a mobile collector to pick up manganese nodules in sea-depth of $5000 \mathrm{~m}$ and more. To guarantee high mobility, the collector is running on crawlers. To analyse their efficiency, several components of the collector, in the first place the pick up mechanism and the crawlers were tested. A further task is the design and development of a controlling and automation system to operate the collector mon the sea bottom. In working areas with difficult and unpredictible surface conditions, which can not be programmed for controlled operation (open loop), a special path control by skid/slip control of the crawler (closed loop) will be provided.

\section{INTRODUCTION}

The worldwide rising demands for raw materials and the foreseeable exhaustion of present deposits require future explorations of pay material even in the deep sea, where giant resources are existing. The exploration of marine resources, with the exception of oil is just at the beginning. One of the most important marine deposits are manganese nodules, which consist of different metals, e.g. manganese, iron, copper, nickel, cobalt and titan.

In 1985 several German and French institutes started to cooperate in developing a manganese nodule mining system. The most important aim is the development and the construction of reliable and non-polluting collectors for manganese nodule mining in sea depth of $5000 \mathrm{~m}$.

\section{MINING SYSTEM}

The entire mining system consists of the following components :

- A vessel serves as floating platform which transports the collector to the operational area. Also the piloting of the collector is done from this vessel.

- The pipe conduit helps transporting the nodules from the sea floor to the vessel.

- The nodules will be conveyed either by pumps or by air-lift techniques.

- A buffer is located at the lower end of the pipe conduit to decouple the hydraulic conveyor systems "pipe conduit" and 
"flexible tube". Furthermore it is possible to integrate a crusher and a batcher in this buffer.

- The flexible hose protects the collector from forces caused by current effecting the pipe conduit.

- The collector itself is the part of the mining system which picks up the nodules from the floor and leads them to the conveyor system. Simultaneously the separation of sediment and oversize nodules is carried out. As a mobile part of the mining system the collector is running on crawlers.

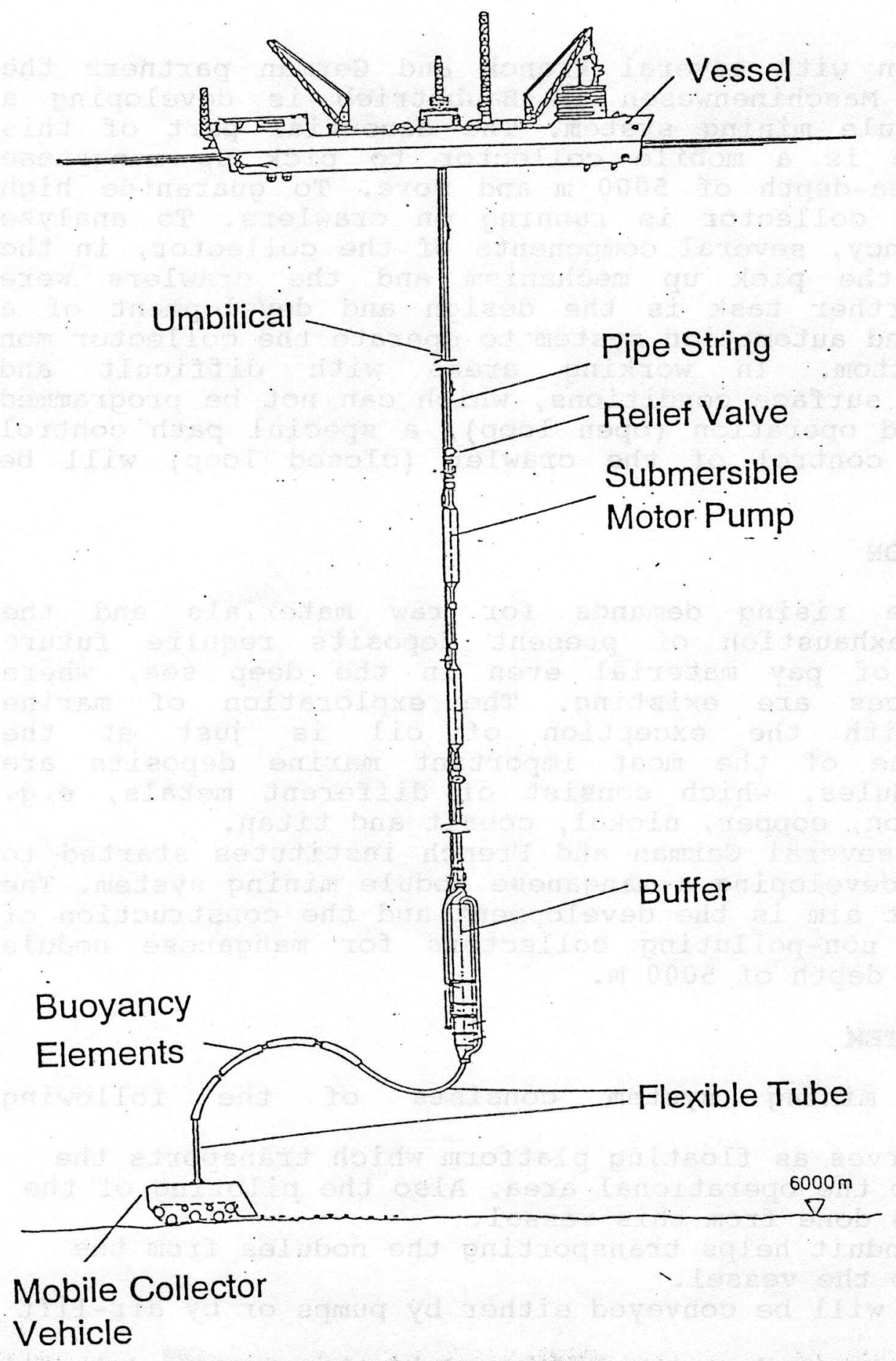




\subsection{COLLECTOR TESTS}

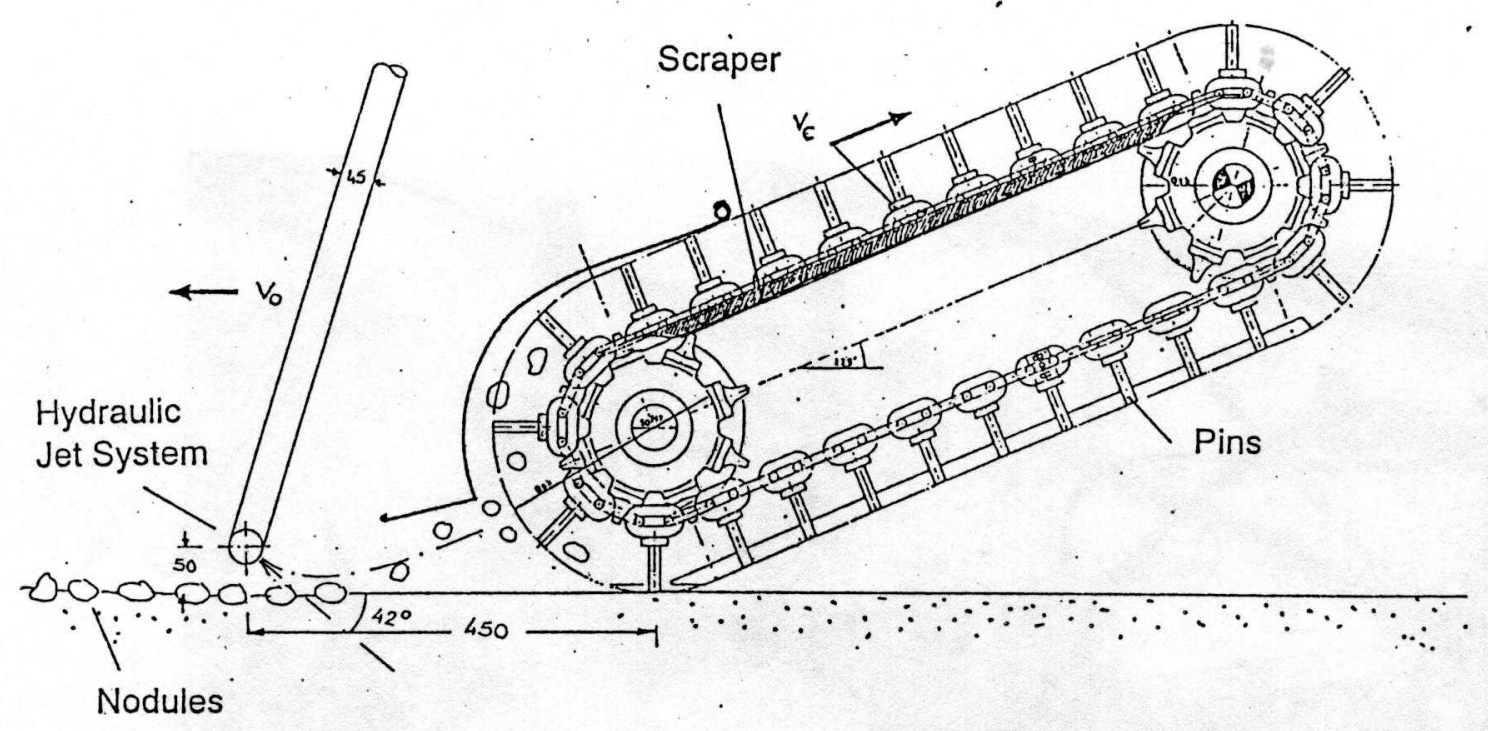

Fig. 2: One of several pick up principles

Three different types of full-scale collectors were tested. The purpose of the test series was:

- Determination of the most qualified collector system and its components by special attention to the internal nodule transportation.

- Verification of structure.

- Investigation of appearing forces.

- Determination of the necessary input power.

- Observation of separation of nodules and soil.

- Investigation of performance by considering morphology of deposits and the distance between collector front-end and sea-floor.

\subsection{SOIL-VEHICLE INTERACTION}

To safely move the collector on the sea-bed, an efficient crawler is necessary. For this purpose extensive experiments with a testing vehicle have been carried out. Both the crawler tests and the preceding collector tests took place in the test channel of the Institut für Maschinenwesen im Baubetrieb. This channel with a length of $63 \mathrm{~m}$ and $6 \mathrm{~m}$ in width meets the requirements for a successful realization of such experiments.

Different types of crawlers and grousers can be assembled to be tested. The soil of the floor consists of a bentonite-water-mixture to simulate deep-sea floor conditions.

To carry out tests, several parameters, e.g. ground pressure, distribution of the vehicle dead weight, 
The goal of these tests is to get detailed information of the driving performance, like sinkage, traction power, velocity and slip of the crawlers and necessary hydraulik pressure of the driving motors.

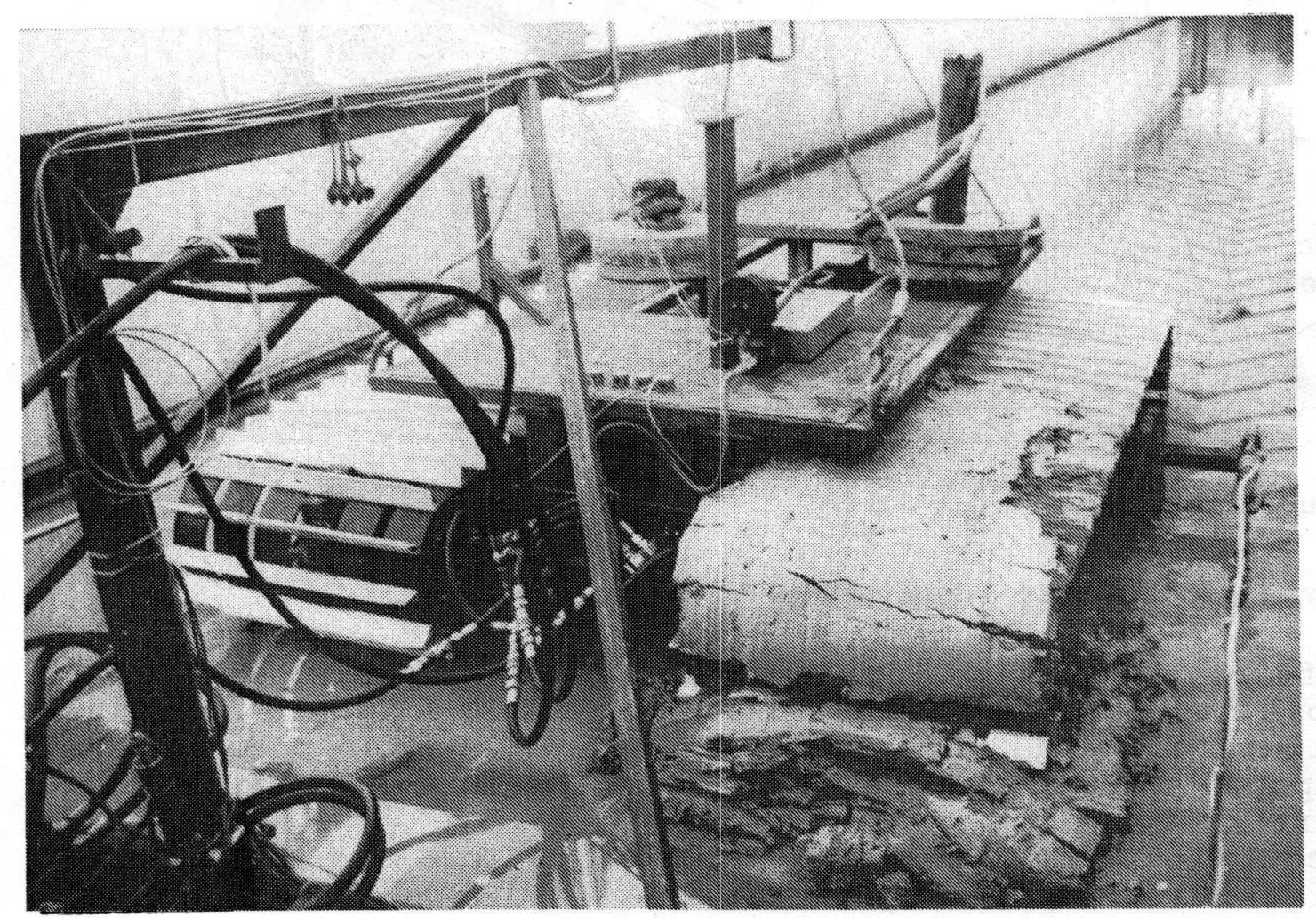

Fig.3: Crawler in test

\section{CONVENTIONAL COLLECTOR CONTROL}

\subsection{MINING STRATEGY}

The mining strategy determines the track of the collector on the sea-floor to pick up the nodules most effectively in the indicated area. In this context a multitude of criteria play an important part, e.g. the yield of the indicated area, track overlapping, parallel steering of several tracks, turns and adaption to topographical conditions.

In practice the route must follow the selected mining strategy. For this purpose an accurate control system is essential. Therefore several concepts have been elaborated.

\subsection{SENSOR APPLICATION CONCEPT}

The suggestion of sensor application for a supervising system 


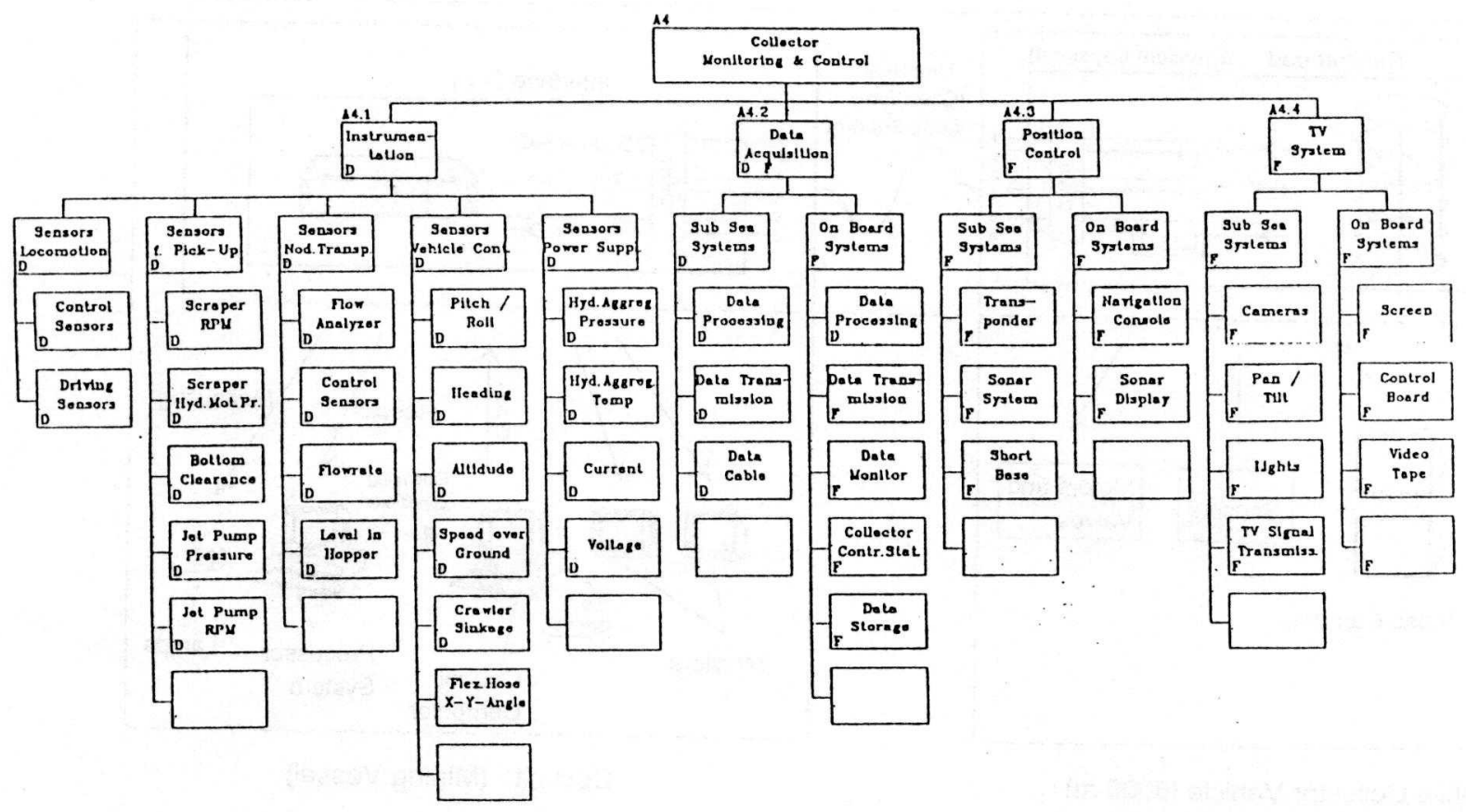

Fig.4: System overview

For steering the collector, two different operating principles are provided: Manual steering by joystick or autopilot steering.

The course of the collector is surveyed by an acoustic positionning system. For this purpose a transponder array is layed out on the sea-floor. Steering by video control should only be practiced exceptionally, because even in clear water the focus of the cameras reaches only 5 to 10 meters.

\subsection{COMMUNICATION AND INTERACTION CONCEPT}

The communication concept provides further automation of the operations of the collector. Because of the complexity of the trafficability on extremely soft soil concerning combinations of longitudinal or lateral inclinations and curves, gradients etc. it is planned to realize an on-line communication system.

The collector is equiped with sensors. Signals are processed by a computer, the so-called sensorhead, which is situated on the collector. These signals are transfered via glass fiber cable to another computer aboard the vessel, the so-called interface.

The interface serves as a link to the control and visualizing system. This system consists of two PCs and several displays. The PCs are used as remote control elements and for processing the measuring results.

The remote control element transfers the orders given by joysticks to the interface. Then the orders are transmitted to the sonsorhead and finally amplified to the actors. 


\section{DATA TRANSMISSION}

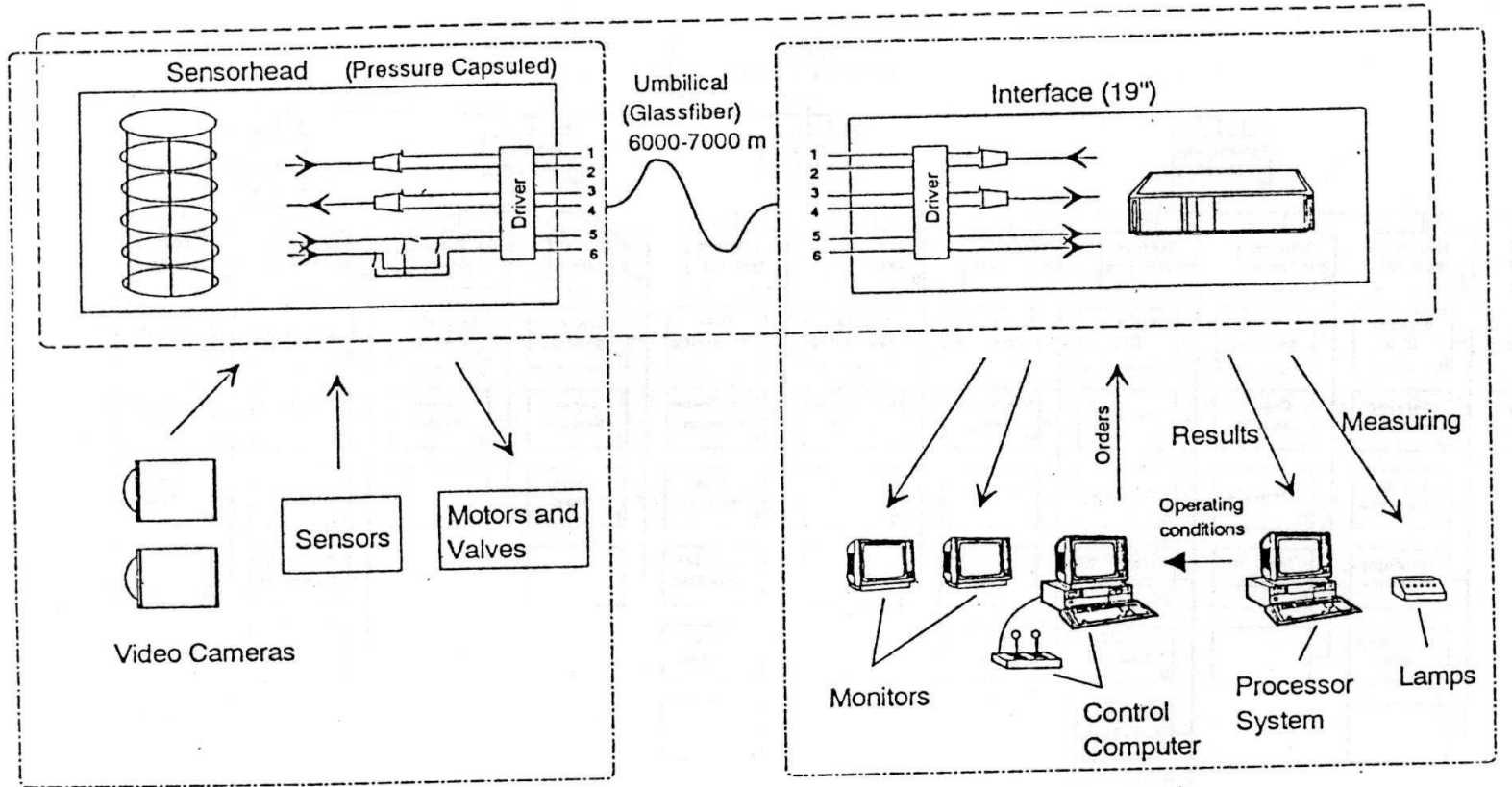

Mobile Collector Vehicle $(6000 \mathrm{~m})$

Cockpit (Mining Vessel)

Fig. 5: Communication System

\section{COLLECTOR AUTOMATION}

\subsection{INITIAL STAGE OF DEVELOPMENT}

According to a proposal of the Institut für Maschinenwesen im Baubetrieb the conventional control system (see chapter 4) of the manipulator will be further developed into a digital control system of the collector. This control system will be based upon an appropriate micro processing unit which is characterized by the following functions:

- Control of the collector

- Freeprogramable trajectories for autonomous mobility within the specified area

- Positioning of collector

- Collision avoiding between collector and rocks or other obstacles

In working areas with difficult surface conditions which are unpredictable and therefore can not be programmed for a controlled operation a special path control by skid/slip control of the crawler will be implemented.

For this purpose the institute will design and develop required algorythms of the collector control system.

\subsection{SYSTEM CONFIGURATION}

For this application it is planned to design a system with distributed intelligence: 
cable network. However the control unit itself will be installed in the collector itself. The onboard control unit will process various sensor data, interpret drive commands, control the vehicle, the crawler skid/slip and ground pressure and finally survey all local functions.

The collector follows the programmed motion commands by user programming. Motion commands are transformed into motion data by RC interpreter. Execution and control is done by data feedback through the RC. These functions are crucial for real time operation and therefore have to be processed by an onboard control unit in real time. Thus a data exchange is required between interpreter and motion generator.

\subsection{COLLECTOR CONTROL}

\subsubsection{CONTROL STRUCTURE AND FUNCTIONS}

The autonomous collector should be capable to partly react to changing conditions of the environment. The path procedures/patterns are preprogrammed in order to avoid down times of the collector. During operation the data generated off-line are processed. The onboard control should realize unexpected obstacles in order to avoid collisions. The collision avoidance function generates an alarm resulting in a shift of the automatic control mode in a remote control mode. Having avoided the obstacle by remote control the system can be reset to the preprogrammed automatic control. Future developments have to consider an automatic sensor based system.

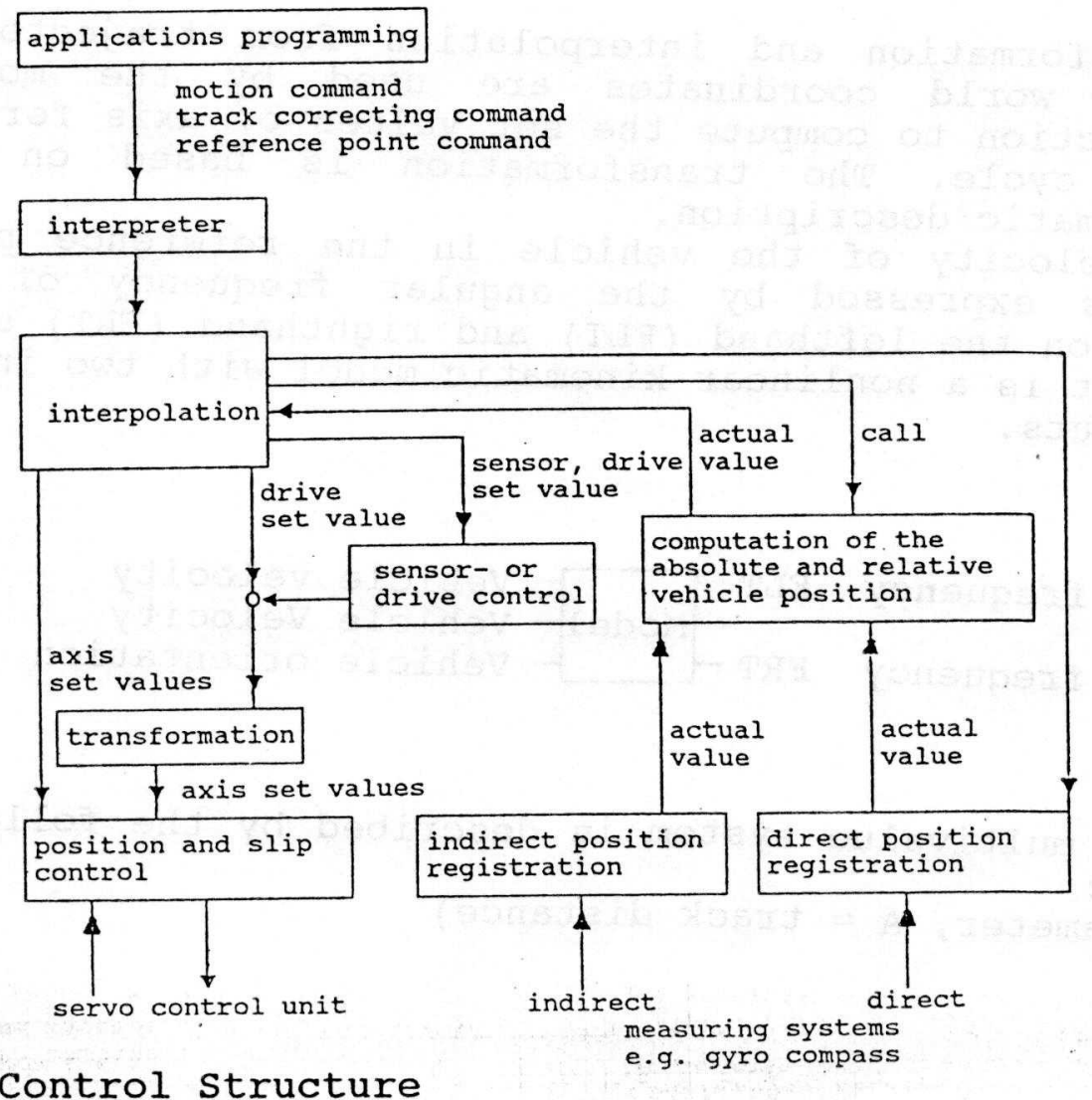

Fig. 6: Control Structure 


\subsubsection{APPLICATION PROGRAMMING}

For user friendly operation the programming method should enable the automatic generation of trajectories from working area boundaries input and also the possibility to describe and control the trajectories in world coordinates rather than vehicle coordinates.

\subsubsection{INTERPRETER}

The interpreter is necessary as a well defined interface between the application programs and the control unit (RC). It initializes the path generation and the process of operation and it supplies them with the needed data.

\subsubsection{INTERPOLATOR}

Following interpolator functions are provided:

- Interpolation in world coordinates

- Interpolation in vehicle coordinates

- Interpolation in line coordinates with additional continious path control to follow a virtually set path

- Path correction in reference points:

-Call of the actual value acquisition

- Calculation of the real actual position

- Output of the newly set values according to the corrected path

- Generation of set values for sensor controlled positioning

\section{$5 \cdot 3 \cdot 5$ TRANSFORMATION}

Backward transformation and interpolation from trajectories given in set world coordinates are used by the motion generation function to compute the set values of axis for the next control cycle. The transformation is based on the following kinematic description.

If the velocity of the vehicle in the reference point XMT, YMT, NMT is expressed by the angular frequency of the crawler drive on the lefthand (FLT) and righthand (FRT) side, then the result is a nonlinear kinematic model with two inputs and three outputs.

$\begin{array}{ll}\text { Drive angular frequency } & \text { FLT } \\ \text { Drive angular frequency } & \text { FRT }\end{array}-\begin{array}{ll}\text { Model- Vehicle velocity } & \text { XMT } \\ \text { Vehicle Velocity } & \text { YMT } \\ \text { NMT }\end{array}$

The nonlinear multivalue system is described by the following block diagram:

( $D=$ wheel diameter, $A=$ track distance) 


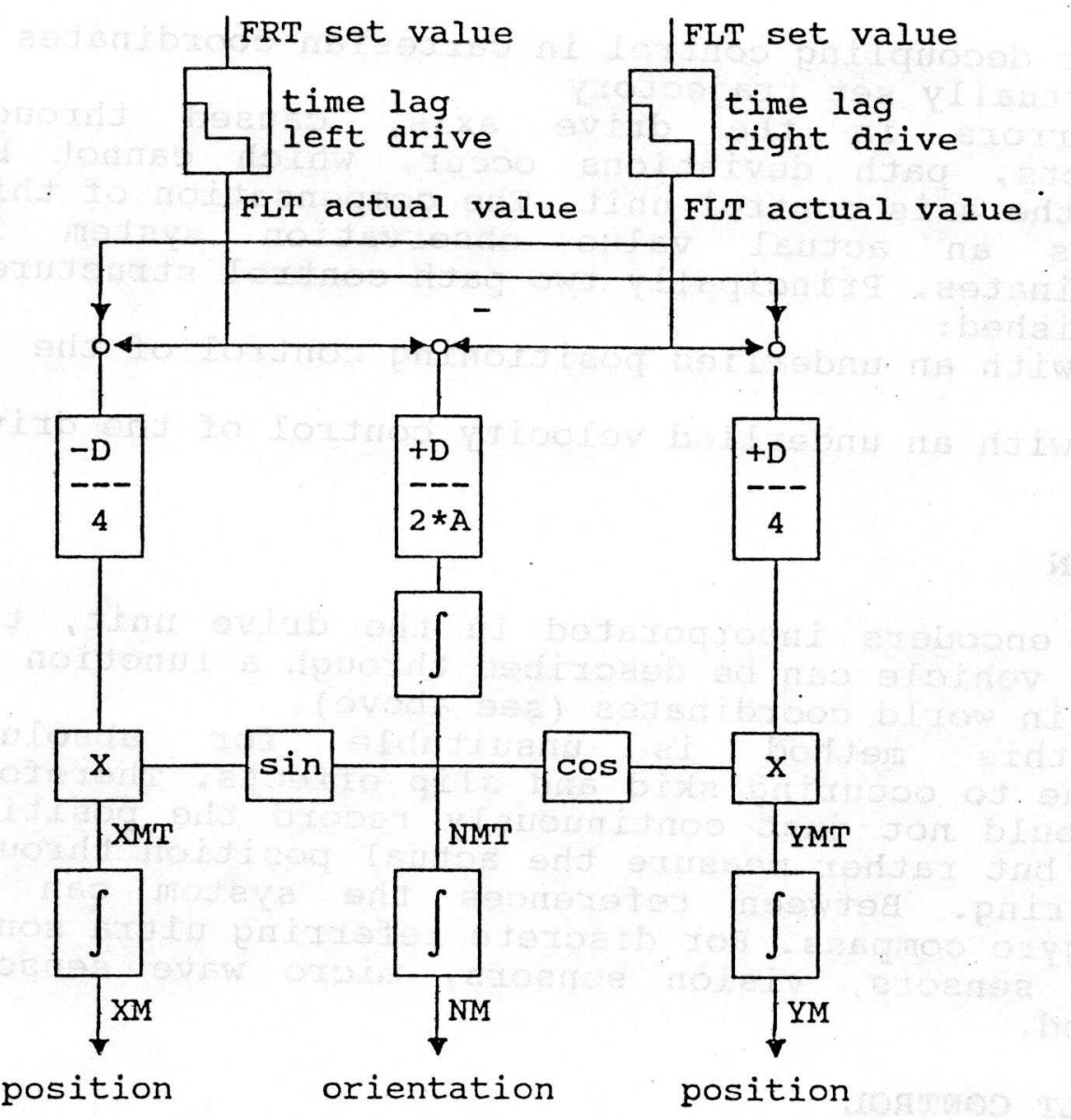

Fig. 7: Block diagram of crawler kinematic

\subsubsection{POSITIONING AND SLIP CONTROL}

The optimal course control would be a system on board of the vehicle compensating the actual deviation caused by local environmental conditions.

Such a system asks for an axis position and slip control including all accessible sensor data which affect the torque of the crawler and its ground pressure in such a way that any skid/slip effect can be compensated and a maximum of power can be transferred.

\subsubsection{SENSOR AND PATH CONTROL WITH CLOSED LOOP}

For guiding around obstacles by sensors it is necessary to acquire sensor data and convert them into cartesian coordinates. According to the features of the closed loop control a nonlinear multivalue system is needed (see above). As an optimal control law like in linear systems is not available, a solution must be approximated by:

- Path control in world coordinates according to the principle of kinematic decoupling and a dependent axis control in vehicle coordinates

- Conventional positioning control with calculation of stable 
- Real nonlinear decoupling control in cartesian coordinates or along a virtually set trajectory

Through errors in the drive axis, caused through skid/slip effects, path deviations occur, which cannot be compensated by the axis control unit. The compensation of this errors requires an actual value observation system in cartesian coordinates. Principally two path control structures can be distinguished:

- Path control with an underlied positioning control of the drive axis

- Path control with an underlied velocity control of the drive axis

\section{3 .8 NAVIGATION}

Through rotary encoders incorporated in the drive unit, the position of the vehicle can be described through a function of angle and time in world coordinates (see above).

However this method is unsuitable for absolute positioning due to occuring skid and slip effects. Therefore the sensors should not just continuously record the position of the vehicle but rather measure the actual position through discrete referring. Between references the system can be controlled by gyro compass. For discrete referring ultra sonic sensors, laser sensors, vision sensors, micro wave sensors etc. can be used.

\subsection{SCRAPER BELT CONTROL}

The speed of the collector is guided by master slave operation in order to avoid material jam in front of the collector and whirling of sediment resulting from excess speed.

The vertical distance of the scraper front end is controlled by feedback from force and torque with the aim to optimize the pick up rate.

\section{FINAL REMARKS}

Research and principle design of the vehicle and the collector attachment have been completed. It has not yet been decided which control system will be implemented and which degree of automation will be finally incorporated. Further research and development is going on.

However, it was found worth-while to present the whole system in the context of this conference as an example for a mining machine, a special type of construction robot in a very hostile and difficult environment. 